\title{
AN F. AND M. RIESZ TYPE THEOREM FOR THE UNIT BALL IN COMPLEX $N$-SPACE
}

\author{
CLINTON KOLASKI 1
}

\begin{abstract}
Let $M_{B}$ denote Lebesgue measure on the open unit ball, $B$, in complex $N$-space and let $M(B)$ denote the space of Borel measures on $B$. The volume Poisson kernel $\chi: \bar{B} \times B \rightarrow(0, \infty)$ is defined and then we prove

THEOREM. If $\mu \in M(B)$ and if $\mu^{\sharp}(w)=\int_{B} \chi(z, w) d \mu(z)$ is pluriharmonic in $B$, then $\mu^{\#} \in L^{\prime}\left(M_{B}\right)$ and $\mu=\mu^{\sharp} \cdot M_{B}$.
\end{abstract}

1.1. Basic definitions and notation. We shall let $V_{N}$ denote $N$-dimensional complex space $\left(\mathbf{C}^{N}\right)$ topologized by the inner product $\langle z, w\rangle=z_{1} \bar{w}_{1}+\cdots$ $+z_{N} \bar{w}_{N}$. The open unit ball in $V_{1}$ is denoted by $D$ (for disc); its boundary is the unit circle $T=\{\langle z, z\rangle=1\}$. For $N \geqslant 2$, the open unit ball shall be denoted by $B$; its boundary is the sphere $S=\{\langle z, z\rangle=1\}$.

For $(z, w) \in \mathbf{C}^{N} \times \mathbf{C}^{N} ; \alpha \in Z_{+}^{N}$ (N-tuple of nonnegative integers) we define $\quad z^{\alpha}=z_{1}^{\alpha_{1}} z_{2}^{\alpha_{2}} \cdots z_{N}^{\alpha_{N}},|\alpha|=\alpha_{1}+\alpha_{2}+\cdots+\alpha_{N} \quad$ and $\quad \alpha !=\left(\alpha_{1} !\right)$ $\cdot\left(\alpha_{2} !\right) \cdots\left(\alpha_{N} !\right)$.

We shall let $M_{T}, M_{S}$ and $M_{B}$ denote Lebesgue measure on $T, S$ and $B$, respectively, all normalized to have unit mass. As is well known, if $E$ is a Borel subset of $S$, then $M_{S}(E)=M_{B}(\tilde{E})$ where $\tilde{E}=\{r z \mid 0 \leqslant r<1, z \in E\}$.

Given a subset $E$ of $V_{N}, C_{0}(E)$ shall denote the class of continuous complex valued functions on $E$ which vanish at infinity. We recall that $C_{0}(E)$ is a Banach space under the supremum norm; its dual (when $E$ is locally compact) is given by $M(E)$, the space of (bounded) complex regular Borel measures on E. by

For $\mu \in M(B)$ and $f \in L^{1}\left(M_{T}\right)$ we define the convolution $\mu * f \in M(B)$

$$
\int_{B} h d(\mu * f)=\int_{T}\left[\int_{B} h(\bar{\omega} z) d \mu(z)\right] f(\omega) d M_{T}(\omega)
$$

for all $h \in C_{0}(B)$.

In this paper we only consider convolutions with the Fejér kernel, $\left\{K_{s}\right\}_{s=0}^{\infty}$, for $T$.

Specifically,

Received by the editors May 12, 1975.

AMS (MOS) subject classifications (1970). Primary 43A32; Secondary 31C10, 32H10, 46G99.

Key words and phrases. Complex measure, Poisson kernel, convolution, pluriharmonic.

1 This work formed part of the author's Ph. D. dissertation which was written under the supervision of Professor F. Forelli at the University of Wisconsin. 


$$
K_{s}(\omega)=\sum_{n=-s}^{s} \hat{K}_{s}(n) \omega^{n}, \quad \text { for all } \omega \in T
$$

where

$$
\tilde{K}_{s}(n)=\int_{T} K_{s}(\omega) \bar{\omega}^{n} d M_{T}(\omega)= \begin{cases}1-|n| /(s+1) & \text { for }|n| \leqslant s \\ 0 & \text { for }|n|>s\end{cases}
$$

1.2. Poisson kernels. The Poisson kernel for the disc is the function $P: \bar{D} \times D \rightarrow(0, \infty)$ defined by $P(z, w)=(1-w \bar{w}) /|1-z \bar{w}|^{2}$. If $\mu \in M(T)$, its Poisson integral is the function defined in $D$ by

$$
P[\mu](w)=\int_{T} P(z, w) d M_{T}(z) .
$$

As is well known, if $f$ is harmonic in a neighborhood containing $\bar{D}$, then $P[f]=f$ in $D$.

Generalizations of the Poisson kernel to a half plane, a ball in real $N$-space and a polydisc in $V_{N}$ can be found in [4], [3] and [5], respectively. The corresponding Poisson integral is defined analogously relative to Lebesgue (area) measure on the line, sphere and torus bounding the respective regions. In each case it is shown that the Poisson integral reproduces harmonic functions ( $N$-harmonic is required in the polydisc case).

A consideration of the action of $G(B)$, the group of holomorphic homeomorphisms on $B$, has led to the discovery of two kernel functions $\beta$ and $\chi: \bar{B} \times B \rightarrow(0, \infty)$ defined by

$$
\beta(z, w)=\left[(1-\langle w, w\rangle) /|1-\langle z, w\rangle|^{2}\right]^{N}
$$

and

$$
\chi^{w}(z) \equiv \chi(z, w)=\left[(1-\langle w, w\rangle) /|1-\langle z, w\rangle|^{2}\right]^{N+1} .
$$

$\beta$ is called the Poisson kernel for $B$ since $\beta=P$ when $N=1$ and, moreover, $\beta M_{S}$ has the significant property of reproducing pluriharmonic functions on $\bar{B}$ [1, Proposition 2.4].

The kernel $\chi$ was discovered by Forelli as follows. Let $f \in L^{1}\left(M_{B}\right)$ be pluriharmonic on $B$ (i.e., the real part of a holomorphic function) and let $\phi \in G(B)$. Using the averaging principle for harmonic functions, we find

$$
f[\phi(0)]=\int_{B}(f \circ \phi)(z) d M_{B}(z)=\int_{B} f(z) \cdot\left|J_{\phi^{-1}}(z)\right| d M_{B}(z)
$$

where $J_{\phi^{-1}}$ is the Jacobian of $\phi^{-1}$.

By using the characterization of $G(B)$ in [1, Proposition 2.2], one may show $\left|J_{\phi^{-1}}(z)\right|=\chi(z, \phi(0))$. Since $G(B)$ acts transitively on $B,(1.4)$ may be written in the form

$$
f(w)=\int_{B} f(z) \chi^{w}(z) d M_{B}(z) .
$$

Thus $\chi$ is a reproducing kernel for the class of $M_{B}$-summable pluriharmonic 
functions in $B$. For this reason $\chi$ is referred to as the (volume) Poisson kernel of $B$.

\subsection{F. and M. Riesz type theorems.}

The Classical F. AND M. Riesz TheOREM. If $\mu \in M(T)$ and if $\int_{T} e^{-i n \theta} d \mu(\theta)$ $=0$ for $n=-1,-2, \ldots$, then $\mu \ll M_{T}$.

Equivalently,

If $\mu \in M(T)$ and if $P[\mu]$ is holomorphic in $D$, then $\mu \ll M_{T}$.

As a consequence of Theorem 3.1, we have the following generalization.

If $\mu \in M(S)$ and if $\beta[\mu](w) \equiv \int_{S} \beta(z, w) d \mu(z)$ is holomorphic in $B$, then $\mu \ll M_{S}$.

Since the kernels $\beta$ and $\chi$ are so similar (both arising from the action of $G(B)$ ) one might naturally ask if an analogous theorem holds for the kernel $\chi$. Theorem 2.3 answers this question affirmatively.

2. An F. and $M$. Riesz type theorem for $B$ relative to $\chi$. Let the map $M(B) \stackrel{\#}{\longrightarrow} C^{\infty}(B)$ be given by

$$
\mu^{\#}(w)=\int_{B} \chi^{w}(z) d \mu(z)
$$

THEOREM 2.1. The map $M(B) \stackrel{\#}{\longrightarrow} C^{\infty}(B)$ is one-to-one.

Proof. Since the map $\mu \rightarrow \mu^{\#}$ is clearly linear, it suffices to show $\mu^{\#}=0$ implies $\mu=0$.

Repeated differentiation of $(1-x)^{-1}=\sum_{n=0}^{\infty} x^{n}$ leads to

$$
(1-x)^{-(N+1)}=\sum_{n=0}^{\infty}\left(\begin{array}{c}
N+n \\
N
\end{array}\right) x^{n}
$$

with convergence holding whenever $|x|<1$.

For a fixed $w \in B$, it follows from (2.2) that

$$
[1-\langle z, w\rangle]^{-(N+1)}=\sum_{n=0}^{\infty}\left(\begin{array}{c}
N+n \\
N
\end{array}\right)\langle z, w\rangle^{n}
$$

with absolute and uniform convergence for all $z \in B$.

Combining identity (2.3) with definitions (1.3) and (2.1) yields

$$
\mu^{\#}(w)=[1-\langle w, w\rangle]^{N+1} \sum_{m, n=0}^{\infty}\left(\begin{array}{c}
N+m \\
N
\end{array}\right)\left(\begin{array}{c}
N+n \\
N
\end{array}\right) \int_{B}\langle z, w\rangle^{m}\langle w, z\rangle^{n} d \mu(z) .
$$

Since $\lambda w \in B$ whenever $\lambda \in D$ and $w \in B$, we find

$$
\mu^{\#}(\lambda w)=[1-\langle\lambda w, \lambda w\rangle]^{N+1}
$$

$$
\cdot \sum_{m, n=0}^{\infty}\left(\begin{array}{c}
N+m \\
N
\end{array}\right)\left(\begin{array}{c}
N+n \\
N
\end{array}\right)\left[\int_{B}\langle z, w\rangle^{m}\langle w, z\rangle^{n} d \mu(z)\right] \cdot \bar{\lambda}^{m} \lambda^{n} .
$$


It follows directly from (2.4) that $\mu^{\#}=0$ implies

$$
\int_{B}\langle z, w\rangle^{m}\langle w, z\rangle^{n} d \mu(z)=0
$$

for all $(m, n) \in Z_{+} \times Z_{+}$and for all $w \in B$.

As a consequence of the well-known multinomial formula, we find

$$
\langle z, w\rangle^{m}=\left(\sum_{K=1}^{N} z_{K} \bar{w}_{K}\right)^{m}=\sum_{|\alpha|=m} \frac{m !}{\alpha !} z^{\alpha} \bar{w}^{\alpha}
$$

for all $m \in Z_{+}$.

It is now clear from (2.5) and (2.6) that

$$
\sum_{|\alpha|=m} \sum_{|\beta|=n}\left(\frac{m !}{\alpha !}\right)\left(\frac{n !}{\beta !}\right)\left[\int_{B} z^{\alpha} \bar{z}^{\beta} d \mu(z)\right] \bar{w}^{\alpha} w^{\beta}=0
$$

for all $(m, n) \in Z_{+} \times Z_{+}$and for all $w \in B$. Hence, $\int_{B} z^{\alpha} \bar{z}^{\beta} d \mu(z)=0$ for all $(\alpha, \beta) \in Z_{+}^{N} \times Z_{+}^{N}$, which implies $\mu=0$ as a consequence of the StoneWeierstrass theorem.

Lemma 2.2. If $\mu^{\#}$ is pluriharmonic in $B$, then $\left(\mu * K_{s}\right)^{\#}$ is a pluriharmonic polynomial.

Proof. By our hypothesis $\mu^{\#}=\operatorname{Re} f$ for some function $f$ holomorphic in $B$. Being holomorphic, $f$ may be written in the form $f=\sum_{k=0}^{\infty} F_{k}$ where each $F_{k}$ is a homogeneous polynomial of degree $k$.

Let us compute $\left(\mu * K_{s}\right)$ \# $(w)$ for a fixed $w$ in $B$. Before proceeding we note that our series for $f$ is absolutely and uniformly convergent on the compact set $w T=\{\omega w \mid \omega \in T\}$ and that $\chi^{w}(\omega z)=\chi^{\bar{\omega} w}(z)$ for all $(z, w) \in B \times B$ and all $\omega \in T$.

Combining the above observations with equation (1.1) yields

$$
\begin{aligned}
\left(\mu * K_{s}\right)^{\#}(w) & =\int_{T}\left[\int_{B} \chi^{\omega w}(z) d \mu(z)\right] K_{s}(\omega) d M_{T}(\omega) \\
& =\int_{T} \mu^{\#}(\omega w) K_{s}(\omega) d M_{T}(\omega) \\
& =\int_{T} \operatorname{Re}\left[\sum_{k=0}^{\infty} F_{k}(w) \omega^{k}\right] K_{s}(\omega) d M_{T}(\omega)=\operatorname{Re} \sum_{k=0}^{s} \hat{K}_{s}(-k) F_{k}(w) .
\end{aligned}
$$

THEOREM 2.3. If $\mu^{\#}$ is pluriharmonic in $B$, then $\mu^{\#} \in L^{1}\left(M_{B}\right)$ and $\mu=\mu^{\#}$ - $M_{B}$.

Proof. Let $h_{s}$ denote the pluriharmonic polynomial $\left(\mu * K_{s}\right)^{\#}$. Since our kernel $\chi$ reproduces such functions (recall equation (1.5)) we find for $w \in B$,

$$
\left(h_{s} \cdot M_{B}\right)^{\#}(w)=\int_{B} \chi^{w} \cdot h_{s} d M_{B}=h_{s}(w)=\left(\mu * K_{s}\right)^{\#}(w) .
$$

Hence,

$$
h_{s} \cdot M_{B}=\mu * K_{s}
$$

as a consequence of Theorem 2.1. 
Using the fact that the Fejér kernel for $T$ is an approximate identity together with the fact that, for a fixed $w \in B, \chi^{w}$ is a bounded continuous function on $B$, we find

$$
\begin{aligned}
\mu^{\#}(w) & =\int_{B} \chi^{w} d \mu=\operatorname{Lim}_{s \rightarrow \infty} \int_{B} \chi^{w} d\left(\mu * K_{s}\right) \\
& =\operatorname{Lim}_{s \rightarrow \infty}\left(\mu * K_{s}\right)^{\#}(w)=\operatorname{Lim}_{s \rightarrow \infty} h_{s}(w) .
\end{aligned}
$$

Combining the results of (2.7) and (2.8) with Fatou's lemma leads to

$$
\begin{aligned}
\int\left|\mu^{\#}\right| d M_{B} & \leqslant \operatorname{Lim}_{s \rightarrow \infty} \operatorname{Inf} \int\left|h_{s}\right| d M_{B} \leqslant \operatorname{Sup}_{s}\left\|h_{s} \cdot M_{B}\right\| \\
& =\operatorname{Sup}_{s}\left\|\mu * K_{s}\right\| \leqslant \operatorname{Sup}_{s}\|\mu\| \cdot \int_{T} K_{s} d M_{T}=\|\mu\|<\infty .
\end{aligned}
$$

Thus $\mu^{\#}$ is an $M_{B^{-}}$-summable pluriharmonic function on $B$. Since $\chi$ reproduces such functions we find

$$
\left(\mu^{\#} \cdot M_{B}\right)^{\#}(w)=\int \chi^{w} \cdot \mu^{\#} d M_{B}=\mu^{\#}(w)
$$

for all $w \in B$. Thus by Theorem 2.1, $\mu=\mu^{\#} \cdot M_{B}$.

\section{A consequence of Theorem 2.3.}

3.1. The spaces $H(\sigma)$ and $\mathcal{H}(\sigma)$. Let $E \subset V_{N}$ be bounded, nonempty, locally compact and satisfy $\omega z \in E$ whenever $(\omega, z) \in T \times E$. Let $0 \neq \sigma \in M(E)$ be $T$-invariant, i.e., $\sigma(\omega A)=\sigma(A)$ for all $\omega \in T$ and all Borel subsets $A$ of $E$. We will denote by $H(\sigma)[\mathcal{H}(\sigma)]$ the $w\left[M(E), C_{0}(E)\right]$ closure of the class of all measures in $M(E)$ of the form $g \sigma[(\operatorname{Re} g) \sigma]$ where $g$ is a polynomial. More explicitly, if $\mu \in H(\sigma)[\nu \in \mathcal{H}(\sigma)]$, if $F \subset C_{0}(E)$ is finite and if $\varepsilon>0$, then there is a polynomial $g(z)=\sum_{|\alpha| \leqslant M} C_{\alpha} z^{\alpha}$ such that

$$
\left|\int f d \mu-\int f g d \sigma\right|<\varepsilon, \quad\left[\left|\int f d \nu-\int f \cdot(\operatorname{Re} g) d \sigma\right|<\varepsilon\right],
$$

for every $f \in F$.

The following theorem is known [2, Theorem 1.2].

THEOREM 3.1. If $\mu \in H(\sigma)$ and if $\varepsilon>0$, then there is a polynomial $g$ such that $\|\mu-g \sigma\|<\varepsilon$. Thus, if $\mu \in H(\sigma)$, then $\mu \ll \sigma$.

3.2. $\mathcal{H}(\sigma)$ in place of $H(\sigma)$. Theorem 3.1 fails if $H(\sigma)$ is replaced by $\mathcal{H}(\sigma)$ (consider $\sigma=M_{T}$ ), but in [1] Forelli proves several results which strongly suggest that this theorem should hold for $\mathcal{H}\left(M_{S}\right)(N \geqslant 2)$ although the answer is not presently known. Utilizing Theorem 2.3 we shall show that the theorem does hold for $\mathcal{H}\left(M_{B}\right)$.

For the purpose of proving Theorem 3.3 we recall the following fact [2, Proposition 3.6].

Lemma 3.2. If $\mu \in \mathcal{H}(\sigma)$, then $\mu * K_{s}=h_{s} \cdot \sigma$ for some pluriharmonic polynomial $h_{s}$. 
It should be remarked that although this lemma is proved for $\mu \in H(\sigma)$, the same proof also works for $\mu \in \mathcal{H}(\sigma)$.

TheOREM 3.3. Let $\sigma=f \cdot M_{B}$ be a T-invariant measure and assume $|f| \geqslant \varepsilon$ $>0$, a.e. $M_{B}$. If $\mu \in \mathcal{H}(\sigma)$, then $(\mu / f)^{\#}$ is pluriharmonic in $B$ and $\mu=(\mu / f)^{\#}$ $\cdot \sigma$.

Proof. From Lemma 3.2, $\mu \in \mathcal{H}(\sigma)$ implies $\mu * K_{s}=h_{s} \cdot \sigma$ for some pluriharmonic polynomial $h_{s}$.

Since $|f| \geqslant \varepsilon>0$, a.e. $M_{B}$, we may assume $|f| \geqslant \varepsilon>0$ everywhere without affecting $\sigma$. Then $f^{-1} \cdot \mu \in M(B)$, which allows us to compute $(\mu / f) * K_{s}$ and $(\mu / f)^{\#}$.

Since $\sigma=f \cdot M_{B}$ is assumed to be $T$-invariant, it follows that for a given $\omega \in T$ we have $f(\omega z)=f(z)$ for $M_{B}$-almost all $z \in B$, and we may assume this to hold everywhere without affecting $\sigma$.

Hence,

$$
\begin{aligned}
\int_{B} F d\left(\frac{\mu}{f} * K_{s}\right) & =\int_{T}\left[\int_{B} F(\bar{\omega} z) \frac{1}{f(\bar{\omega} z)} d \mu(z)\right] K_{s}(\omega) d M_{T}(\omega) \\
& =\int_{B}\left(\frac{F}{f}\right) d\left(\mu * K_{s}\right)=\int_{B} F \cdot h_{s} d M_{B}
\end{aligned}
$$

for all $F \in C_{0}(B)$, hence

$$
\mu / f * K_{s}=h_{s} \cdot M_{B}
$$

for all $s \in Z_{+}$.

From equation (3.1) it now follows (as in the proof of Theorem 2.3) that for $w \in B$, we have

$$
(\mu / f)^{\#}(w)=\operatorname{Lim}_{s \rightarrow \infty} \int_{B} \chi^{w} d\left(\frac{\mu}{f} * K_{s}\right)=\operatorname{Lim}_{s \rightarrow \infty} \int_{B} \chi^{w} \cdot h_{s} d M_{B}=\operatorname{Lim}_{s \rightarrow \infty} h_{s}(w) .
$$

To see that $(\mu / f)^{\#}$ is pluriharmonic in $B$, it now suffices to show that our sequence $\left\{h_{s}\right\}_{s=0}^{\infty}$ is uniformly bounded on compact subsets of $B$.

If $A$ denotes an arbitrary compact subset of $B$, then

$$
\operatorname{Sup}_{z \in B ; w \in A} \chi^{w}(z)=\operatorname{Sup}_{z \in B ; w \in A}\left[\frac{1-\langle w, w\rangle}{|1-\langle z, w\rangle|^{2}}\right]^{N+1}=M_{A}<\infty .
$$

Hence, for $w \in A$, we find

$$
\begin{aligned}
\left|h_{s}(w)\right| & =\left|\int_{B} \chi^{w} \cdot h_{s} d M_{B}\right|=\left|\int_{B} \chi^{w} d\left(\frac{\mu}{f} * K_{s}\right)\right| \\
& \leqslant \int_{B} \chi^{w} d\left|\frac{\mu}{f} * K_{s}\right| \leqslant M_{A} \cdot\left\|\frac{\mu}{f} * K_{s}\right\| \leqslant M_{A}\left\|\frac{\mu}{f}\right\|
\end{aligned}
$$

which implies

$$
\operatorname{Sup}_{s \in Z_{+} ; w \in A}\left|h_{s}(w)\right| \leqslant M_{A} \cdot\|\mu / f\|<\infty
$$


Thus $(\mu / f)^{\#}$ is pluriharmonic on $B$ so we may conclude from Theorem 2.3 that $\mu / f=(\mu / f)^{\#} \cdot M_{B}$.

\section{BIBLIOGRAPHY}

1. Frank Forelli, Measures whose Poisson integrals are pluriharmonic, Illinois J. Math. 18 (1974), 373-388. MR 49 \#7468.

2. - The theorems of F. and M. Riesz for circular sets, Math. Scand. 33 (1973), 145-152. MR 48 \#5851.

3. L. L. Helms, Introduction to potential theory, Pure and Appl. Math., vol. 22, Wiley, New York, 1969. MR 41 \#5638.

4. Kenneth Hoffman, Banach spaces of analytic functions, Prentice-Hall Ser. in Modern Analysis, Prentice-Hall, Englewood Cliffs, N. J., 1962. MR 24 \# A2844.

5. Walter Rudin, Function theory in polydiscs, Benjamin, New York, 1969. MR 41 \#501.

Department of Mathematics, University of Texas, San Antonio, Texas 78285 